517.957

doi:10.13108/2018-10-1-14

UDC 517.957

\title{
UNSOLVABILITY CONDITIONS FOR SOME INEQUALITIES AND SYSTEMS WITH FUNCTIONAL PARAMETERS AND SINGULAR COEFFICIENTS ON BOUNDARY
}

\author{
E.I. GALAKHOV, O.A. SALIEVA
}

\begin{abstract}
We consider the problem on nonexistence of positive solutions for some nonlinear elliptic inequalities in a bounded domain. The principal parts of the considered inequalities are $p(x)$-Laplacians with variable exponents. The lower terms of the considered inequalities can depend both on the unknown function and its gradient. We assume that the coefficients at the lower terms have singularities at the boundary. To the best of the authors' knowledge, the conditions for nonexistence of solutions to inequalities with variable exponents were not considered before.

We obtain the sufficient conditions for nonexistence of positive solutions in terms of the exponent $p(x)$, of the singularities order and of parameters in the problem. To prove the obtained conditions, we employ an original modification of the nonlinear capacity method proposed by S.I. Pokhozhaev. The method is based on a special choice of test functions in the generalized formulation of the problem and on algebraic transformations of the obtained expression. This allows us to obtain asymptotically sharp apriori estimates for the solutions leading to a contradiction under a certain choice of the parameters. This implies the nonexistence of the solutions. We generalize the obtained results for the case of nonlinear systems with similar conditions for the operators and coefficients.
\end{abstract}

Keywords: elliptic inequalities, variable exponents, nonexistence of solutions, singular coefficients.

Mathematics Subject Classification: 35J60, 35K55, 35R55

\section{INTRODUCTION}

Sufficient conditions for nonexistence of solutions to nonlinear elliptic equations, inequalities and the systems of them was considered by many authors.

The first results for the Laplace operator with a point singularity inside a domain were obtained by H. Brezis and X. Cabré [1] by means of the comparison principle.

S.I. Pokhozhaev proposed the nonlinear capacity method [9] for higher order operators not satisfying the comparison principle. Later this method was developed in joint works with E. Mitidieri and other authors, see monograph [8] and the references therein. This method allowed them to obtain a series of new sharp sufficient unsolvability conditions for nonlinear partial differential inequalities in various functional classes. The method was based on obtaining asymptotically optimal apriori estimates by the algebraic analysis of the integral form of the considered inequality under a special choice of the test functions. The application of this

E.I. Galakhov, O.A. Salieva, Unsolvability Conditions for some inequalities and Systems WITH FUNCTIONAL PARAMETERS AND SINGULAR COEFFICIENTS ON BOUNDARY.

(C) E.I. Galakhov, O.A. Salieva. 2018.

The Work is Supported By the Ministry of Education and Science of Russian Federation (AGREEMENT 05.Y09.21.0013, MAY 19, 2017.).

Submitted December 28, 2016. 
method to various types of elliptic equations and systems can be found, for instance, in [2], [3], [4], 7].

In the present work we employ a modification of the nonlinear capacity method for obtaining sufficient unsolvability conditions for some nonlinear elliptic inequalities in a bounded domain with variable exponents and coefficients with singularities at the boundary. To the best of the authors' knowledge, earlier the unsolvability conditions for inequalities with variable exponents were not considered.

To prove the nonexistence of solutions by the nonlinear capacity method, we construct test functions with various geometric structure taking into account a specific character of the considered problem. Our first results in this direction were published in [5], [6].

The rest of paper consists of two section. In Section 2 we obtain the results on nonexistence of solutions to scalar nonlinear elliptic inequalities, while in Section 3 we do the same for the systems of such inequalities.

Remark on notations: hereinafter the symbol $c$ stands for various positive constants, which can depend on the parameters of the considered problems.

\section{Scalar inequalities}

We consider the problem

$$
\begin{cases}-\operatorname{div}\left(|D u|^{p(x)-2} D u\right) \geqslant \rho^{-\alpha}(x) u^{q(x)}|D u|^{s(x)}, & x \in \Omega, \\ u(x) \geqslant 0, & x \in \Omega,\end{cases}
$$

where $\Omega$ is a bounded domain with a smooth boundary, $p(x), q(x), s(x) \in C(\Omega)$ are functions with a positive infimum, $\rho(x)=\operatorname{dist}(x, \partial \Omega), \alpha \in \mathbb{R}$.

The solutions of problem (1) are treated in the weak sense (in the sense of distributions) according to the following definition.

Definition 2.1. A non-negative function $u \in W_{\mathrm{loc}}^{1, p(x)}(\Omega)$ is called a weak solution (in the sense of distributions) to problem (1) if $\rho^{-\alpha}(x) u^{q(x)}|D u|^{s(x)} \in L_{\text {loc }}^{1}(\Omega)$ and for each nonnegative test function $\psi \in C_{0}^{1}(\Omega)$ the inequality

$$
\int_{\Omega}|D u|^{p(x)-2}(D u, D \psi) d x \geqslant \int_{\Omega} \rho^{-\alpha}(x) u^{q(x)}|D u|^{s(x)} \psi d x
$$

holds.

Remark 2.1. Similarly to [8] we can show that if such solution exists and it is strictly positive in $\Omega$, then (2) holds also for test functions of form $\psi=u^{\gamma} \varphi$ with $\gamma \in \mathbb{R}$ and $\varphi \in C_{0}^{1}(\Omega)$. If $u$ vanishes somewhere in $\Omega$ and $\gamma<0$, we can use the test functions $\psi=(u+\delta)^{\gamma} \varphi$ and pass to the limit as $\delta \rightarrow 0_{+}$. This leads to the same results as in the previous case. This is why in what follows we assume that $u>0$ if this exists.

We introduce the notation

$$
\Omega_{k \eta}=\{x \in \Omega: \rho(x) \geqslant k \eta\} \quad(\eta>0, k=1,2) .
$$

Assume that

$$
\inf _{x \in \Omega} p(x)>1, \quad \inf _{x \in \Omega}(q(x)-p(x))>1
$$


We denote

$$
\begin{aligned}
& b_{\gamma}(x)=\frac{p(x)(q(x)+\gamma)-s(x)(\gamma-1)}{q(x)+s(x)-p(x)+1}, \\
& c_{\gamma}(x)=\frac{p(x)+\gamma-1}{q(x)+s(x)-p(x)+1}, \\
& D(\gamma, \eta)=\int_{\Omega_{\eta} \backslash \Omega_{2 \eta}} \eta^{b_{\gamma}(x)} \rho^{-\alpha c_{\gamma}(x)} d x .
\end{aligned}
$$

Then the following theorem holds.

Theorem 2.1. Assume that there exists $\gamma_{0}<0$ such that for $\gamma \in\left(\gamma_{0}, 0\right)$ we have

$$
\lim _{\eta \rightarrow 0_{+}} D(\gamma, \eta)=0
$$

Then inequality (1) has no non-trivial solutions.

Example 2.1. Let $\Omega=B_{1}(0), p(x) \equiv p=$ const, $q(x) \equiv q=$ const, $s(x) \equiv 0$. Then $\rho(x)=1-|x|$ and inequality (1) becomes

$$
-\Delta_{p} u \geqslant u^{q}(1-|x|)^{-\alpha} \quad\left(x \in B_{1}(0)\right),
$$

while condition (4) holds exactly as $\alpha \geqslant q+1$. It is easy to see that once this condition fails, that is, as $\alpha<q+1$, inequality (5) has a solution of form $C(1-|x|)^{\frac{\alpha-p}{q-p+1}}$ with an appropriate constant $C=C(p, q, \alpha)>0$, that is, the obtained unsolvability condition for inequality (5) is optimal.

Proof. Assume that there exists a non-trivial solution $u$ of inequality (1). We introduce the family of functions $\varphi_{\eta} \in C_{0}^{1}(\Omega ;[0,1])$ of form $\varphi_{\eta}(x)=\xi_{\eta}^{\lambda}(x)$ with

$$
\begin{aligned}
& \xi_{\eta}(x)= \begin{cases}1 & x \in \Omega_{2 \eta}, \\
0 & x \notin \Omega_{\eta},\end{cases} \\
& \left|D \xi_{\eta}(x)\right| \leqslant c \eta^{-1} \quad(x \in \Omega)
\end{aligned}
$$

and with a sufficiently large $\lambda>0$. Then we obtain

$$
\begin{aligned}
\int_{\Omega} \rho^{-\alpha}(x) u^{q(x)+\gamma}|D u|^{s(x)} \varphi_{\eta} d x & \leqslant \int_{\Omega}\left(|D u|^{p(x)-2} D u, D\left(u^{\gamma} \varphi_{\eta}\right)\right) d x \\
& =\gamma \int_{\Omega} u^{\gamma-1}|D u|^{p(x)} \varphi_{\eta} d x+\int_{\Omega} u^{\gamma}|D u|^{p(x)-2}\left(D u, D \varphi_{\eta}\right) d x \\
& \leqslant \gamma \int_{\Omega} u^{\gamma-1}|D u|^{p(x)} \varphi_{\eta} d x+\int_{\Omega} u^{\gamma}|D u|^{p(x)-1}\left|D \varphi_{\eta}\right| d x,
\end{aligned}
$$

which implies

$$
\int_{\Omega} \rho^{-\alpha}(x) u^{q(x)+\gamma}|D u|^{s(x)} \varphi_{\eta} d x+|\gamma| \int_{\Omega} u^{\gamma-1}|D u|^{p(x)} \varphi_{\eta} d x \leqslant \int_{\Omega} u^{\gamma}|D u|^{p(x)-1}\left|D \varphi_{\eta}\right| d x .
$$

We represent the integrand in the right hand side of this inequality as

$$
2^{-\frac{y(x)}{s(x)}} u^{\frac{(q(x)+\gamma) y(x)}{s(x)}}|D u|^{y(x)} \rho^{-\frac{\alpha y(x)}{s(x)}} \varphi_{\eta}^{\frac{y(x)}{s(x)}} \cdot 2^{\frac{y(x)}{s(x)}} u^{\frac{\gamma s(x)-(q(x)+\gamma) y(x)}{s(x)}}|D u|^{p(x)-1-y(x)}\left|D \varphi_{\eta}\right| \cdot \rho^{\frac{\alpha y(x)}{s(x)}} \varphi_{\eta}^{-\frac{y(x)}{s(x)}},
$$

where $y(x)$ will be chosen later. We apply the parametric Young inequality with the exponent $s(x) / y(x)$; we shall show later that $s(x) / y(x)>1$ under an appropriate choice of $y(x)$. This 
yields

$$
\begin{aligned}
& \frac{1}{2} \int_{\Omega} \rho^{-\alpha}(x) u^{q(x)+\gamma}|D u|^{s(x)} \varphi_{\eta} d x+|\gamma| \int_{\Omega} u^{\gamma-1}|D u|^{p(x)} \varphi_{\eta} d x \\
& \quad \leqslant c \int_{\Omega} u^{\frac{\gamma s(x)-(q(x)+\gamma) y(x)}{s(x)-y(x)}}|D u|^{\frac{(p(x)-1-y(x)) s(x)}{s(x)-y(x)}}\left|D \varphi_{\eta}\right|^{\frac{s(x)}{s(x)-y(x)}} \cdot \rho^{\frac{\alpha y(x)}{s(x)-y(x)}} \varphi_{\eta}^{-\frac{y(x)}{s(x)-y(x)}} d x .
\end{aligned}
$$

We apply the Young inequality with the exponent $z(x)$ once again:

$$
\begin{aligned}
& c \int_{\Omega} u^{\frac{\gamma s(x)-(q(x)+\gamma) y(x)}{s(x)-y(x)}}|D u|^{\frac{(p(x)-1-y(x)) s(x)}{s(x)-y(x)}}\left|D \varphi_{\eta}\right|^{\frac{s(x)}{s(x)-y(x)}} \cdot \rho^{\frac{\alpha y(x)}{s(x)-y(x)} \varphi_{\eta}^{-\frac{y(x)}{s(x)-y(x)}} d x} \\
& \leqslant \frac{|\gamma|}{2} \int_{\Omega} u^{\frac{(\gamma s(x)-(q(x)+\gamma) y(x)) z(x)}{s(x)-y(x)}}|D u|^{\frac{(p(x)-1-y(x)) s(x) z(x)}{s(x)-y(x)}} \varphi_{\eta} d x \\
& \quad+c \int_{\Omega}\left|D \varphi_{\eta}\right|^{\frac{s(x) z^{\prime}(x)}{s(x)-y(x)}} \cdot \rho^{\frac{\alpha y(x) z^{\prime}(x)}{s(x)-y(x)}} \varphi_{\eta}^{1-\frac{s(x) z^{\prime}(x)}{s(x)-y(x)}} d x,
\end{aligned}
$$

where

$$
\frac{1}{z(x)}+\frac{1}{z^{\prime}(x)}=1
$$

We choose $y(x)$ and $z(x)$ so that

$$
\left\{\begin{array}{l}
(p(x)-1-y(x)) s(x) z(x)=p(x)(s(x)-y(x)), \\
\frac{\gamma s(x)-(q(x)+\gamma) y(x)}{s(x)-y(x)} \cdot z(x)=\gamma-1,
\end{array}\right.
$$

that is,

$$
\left\{\begin{array}{l}
y(x)=y_{\gamma}(x)=\frac{s(x)(p(x)+\gamma-1)}{p(x)(q(x)+\gamma)-s(x)(\gamma-1)}, \\
z(x)=z_{\gamma}(x)=\frac{p(x)[p(x)(q(x)+\gamma)-s(x)(\gamma-1)-(p(x)+\gamma-1)]}{(p(x)-1)(p(x)(q(x)+\gamma)-s(x)(\gamma-1))-s(x)(p(x)+\gamma-1)} .
\end{array}\right.
$$

We note that as $\gamma=0$, by our assumptions on $q(x), p(x)$ and $s(x)$, for each $x \in \Omega$ we have

$$
\frac{s(x)}{y_{0}(x)}=\frac{p(x) q(x)+s(x)}{p(x)-1}>\frac{p(x) q(x)+s(x)}{q(x)}=p(x)+\frac{s(x)}{q(x)}>p(x)>1
$$

and

$$
z_{0}(x)=\frac{p(x)(q(x)-1)+s(x)+1}{p(x)(q(x)-1)}=1+\frac{s(x)+1}{p(x)(q(x)-1)}>1 .
$$

By the continuity, for sufficiently small $|\gamma|$ this yields: $\frac{s(x)}{y_{\gamma}(x)}>1$ and $z_{\gamma}(x)>1$ for all $x \in \Omega$ and this is needed to apply the Young inequality.

For such $y(x)$ and $z(x)$ and for $\varphi_{\eta}$ with properties (6), (7) and for sufficiently large $\lambda>0$, it follows from (8) that

$$
\frac{1}{2} \int_{\Omega} \rho^{-\alpha}(x) u^{q(x)+\gamma}|D u|^{s(x)} \varphi_{\eta} d x+\frac{|\gamma|}{2} \int_{\Omega} u^{\gamma-1}|D u|^{p(x)} \varphi_{\eta} d x \leqslant c D(\gamma, \eta) .
$$

Passing to the limit as $\eta \rightarrow+0$, by (4) for $\gamma \in\left(\gamma_{0}, 0\right)$ we obtain the contradiction to the assumed nontriviality of $u$ and this completes the proof. 


\section{Systems OF INEQUALITIES}

We consider the system of inequalities

$$
\begin{cases}-\operatorname{div}\left(|D u|^{p(x)-2} D u\right) \geqslant \rho^{-\alpha}(x) v^{q_{1}(x)}|D v|^{q_{2}(x)}, & x \in \Omega, \\ -\operatorname{div}\left(|D v|^{q(x)-2} D v\right) \geqslant \rho^{-\beta}(x) u^{p_{1}(x)}|D u|^{p_{2}(x)}, & x \in \Omega, \\ u, v \geqslant 0, & x \in \Omega,\end{cases}
$$

where $\Omega$ is a bounded domain with a smooth boundary.

We assume that $p, q, p_{1}, q_{1}, p_{2}, q_{2} \in C(\Omega)$ are functions with a positive infimum, $\alpha, \beta \in \mathbb{R}$.

The solutions of system (9) are treated in the weak sense (in the sense of distributions) according to the following definition.

Definition 3.1. A pair of non-negative functions $(u, v) \in W_{\mathrm{loc}}^{1, p}(\Omega) \cap W_{\mathrm{loc}}^{1, q}(\Omega)$ is called a weak solution (in the sense of distributions) to system $(9)$ if $\rho^{-\alpha}(x) v^{q_{1}(x)}|D v|^{q_{2}(x)} \in L_{\mathrm{loc}}^{1}(\Omega)$, $\rho^{-\beta}(x) u^{p_{1}(x)}|D v|^{p_{2}(x)} \in L_{\text {loc }}^{1}(\Omega)$ and for each non-negative test functions $\psi_{1}, \psi_{2}(x) \in C_{0}^{1}(\Omega)$ the inequalities

$$
\begin{aligned}
& \int_{\Omega}|D u|^{p(x)-2}\left(D u, D \psi_{1}\right) d x \geqslant \int_{\Omega} \rho^{-\alpha}(x) v^{q_{1}(x)}|D v|^{q_{2}(x)} \psi_{1} d x, \\
& \int_{\Omega}|D v|^{q(x)-2}\left(D v, D \psi_{2}(x)\right) d x \geqslant \int_{\Omega} \rho^{-\beta}(x) u^{p_{1}(x)}|D u|^{p_{2}(x)} \psi_{2}(x) d x
\end{aligned}
$$

hold.

Remark 3.1. Similarly to Remark 2.1, we can assume that $u>0$ and $v>0$ if they exist and we can employ the test functions $\psi_{1}=u^{\gamma} \varphi \quad \psi_{2}(x)=v^{\gamma} \varphi$ with $\varphi \in C_{0}^{1}(\Omega)$.

We denote

$$
\begin{aligned}
& c_{1, \gamma}(x)=-\frac{q(x)+\gamma-1}{q_{1}(x)+q_{2}(x)-q(x)-\gamma+1}, \quad c_{2, \gamma}(x)=-\frac{(p(x)-1)(1-\gamma)}{p_{1}(x)+\left(p_{2}(x)-p(x)+1\right)(1-\gamma)}, \\
& c_{3, \gamma}(x)=-\frac{q(x)+\gamma-1}{q_{1}(x)+q_{2}(x)-q(x)-\gamma+1}, \quad c_{4, \gamma}(x)=-\frac{(q(x)-1)(1-\gamma)}{q_{1}(x)+\left(q_{2}(x)-q(x)+1\right)(1-\gamma)}, \\
& d_{1, \gamma}(x)=\frac{p(x) p_{1}(x)+p_{2}(x)(1-\gamma)}{p_{1}(x)+p_{2}(x)-p(x)-\gamma+1}, \quad d_{2, \gamma}(x)=\frac{p(x) p_{1}(x)+p_{2}(x)(1-\gamma)}{p_{1}(x)+\left(p_{2}(x)-p(x)+1\right)(1-\gamma)}, \\
& d_{3, \gamma}(x)=\frac{q(x) q_{1}(x)+q_{2}(x)(1-\gamma)}{q_{1}(x)+q_{2}(x)-q(x)-\gamma+1}, \quad d_{4, \gamma}(x)=\frac{q(x) q_{1}(x)+q_{2}(x)(1-\gamma)}{q_{1}(x)+\left(q_{2}(x)-q(x)+1\right)(1-\gamma)}, \\
& D_{j, \gamma}(\eta)=\int_{\Omega_{\eta} \backslash \Omega_{2 \eta}} b^{c_{j, \gamma}(x)}(x) \cdot \eta^{d_{j, \gamma}(x)} d x, \quad j=1,2, \\
& D_{j, \gamma}(\eta)=\int_{\Omega_{\eta} \backslash \Omega_{2 \eta}} a^{c_{j, \gamma}(x)}(x) \cdot \eta^{d_{j, \gamma}(x)} d x, \quad j=3,4 .
\end{aligned}
$$

Then the following theorem holds.

Theorem 3.1. Assume that

$\inf _{x \in \Omega} p(x)>1, \quad \inf _{x \in \Omega} q(x)>1, \quad \inf _{x \in \Omega}\left(p_{1}(x)+p_{2}(x)-p(x)\right)>1, \quad \inf _{x \in \Omega}\left(q_{1}(x)+q_{2}(x)-q(x)\right)>1$ and there exists $\gamma_{0}<0$ such that

$$
\lim _{\eta \rightarrow 0_{+}} D_{j, \gamma}(\eta)=0, \quad j=1, \ldots, 4
$$

for $\gamma \in\left(\gamma_{0}, 0\right)$. Then system (9) has no nontrivial solutions. 
Proof. Let $(u, v)$ be a non-trivial solution to system $(9)$, and $\varphi_{\eta} \in C_{0}^{\infty}(\Omega ;[0,1])$ be test functions of the same form as in the proof of Theorem 2.1 satisfying (6) and (7).

Employing the test function $\psi_{1}(x)=u^{\gamma}(x) \varphi_{\eta}(x)$ in the first inequality in (10) and the function $\psi_{2}(x)=v^{\gamma}(x) \varphi_{\eta}(x)$ in the second inequality, where the number $\gamma$ is such that

$$
\max \left(\inf _{x \in \Omega}(1-p(x)), \inf _{x \in \Omega}(1-q(x)), \gamma_{0}\right)<\gamma<0,
$$

we get

$$
\begin{aligned}
& \int \rho^{-\alpha}(x) v^{q_{1}(x)}|D v|^{q_{2}(x)} u^{\gamma} \varphi_{\eta} d x \leqslant \gamma \int u^{\gamma-1}|D u|^{p(x)} \varphi_{\eta} d x+\int u^{\gamma}|D u|^{p(x)-1}\left|D \varphi_{\eta}\right| d x, \\
& \int \rho^{-\beta}(x) u^{p_{1}(x)}|D u|^{p_{2}(x)} v^{\gamma} \varphi_{\eta} d x \leqslant \gamma \int v^{\gamma-1}|D v|^{q(x)} \varphi_{\eta} d x+\int v^{\gamma}|D v|^{q(x)-1}\left|D \varphi_{\eta}\right| d x .
\end{aligned}
$$

We employ the representation

$$
\begin{aligned}
& u^{\gamma}|D u|^{p(x)-1}=u^{a_{1}(x)}|D u|^{b_{1}(x)} \varphi_{\eta}^{\frac{1}{c_{1}(x)}} u^{\gamma-a_{1}(x)}|D u|^{p(x)-1-b_{1}(x)} \varphi_{\eta}^{-\frac{1}{c_{1}(x)}}, \\
& v^{\gamma}|D v|^{q(x)-1}=v^{a_{2}(x)}|D u|^{b_{2}(x)} \varphi_{\eta}^{\frac{1}{c_{2}(x)}} v^{\gamma-a_{2}(x)}|D v|^{q(x)-1-b_{2}(x)} \varphi_{\eta}^{-\frac{1}{c_{2}(x)}},
\end{aligned}
$$

and apply the parametric Young inequality to the right hand sides in (12) and (13) with the exponents denoted respectively $c_{1}(x)$ and $c_{2}(x)$. We choose the parameters so that

$$
\begin{aligned}
& \left\{\begin{array}{l}
a_{1}(x) c_{1}(x)=\gamma-1, \\
b_{1}(x) c_{1}(x)=p(x), \\
\frac{\gamma-a_{1}(x)}{p(x)-1-b_{1}(x)}=\frac{p_{1}(x)}{p_{2}(x)},
\end{array}\right. \\
& \left\{\begin{array}{l}
a_{2}(x) c_{2}(x)=\gamma-1, \\
b_{2}(x) c_{2}(x)=q(x), \\
\frac{\gamma-a_{2}(x)}{q(x)-1-b_{2}(x)}=\frac{q_{1}(x)}{q_{2}(x)} .
\end{array}\right.
\end{aligned}
$$

Remark 3.2. The meaning of the above choice is the preparation to the following use of the Hölder inequality; under appropriate choice of the parameters, we want to obtain $\int \rho^{-\beta}(x) u^{p_{1}(x)}|D u|^{p_{2}(x)} \varphi_{\eta} d x$ and $\int \rho^{-\alpha}(x) v^{q_{1}(x)}|D v|^{q_{2}(x)} \varphi_{\eta} d x$ in the right hand sides of the inequalities.

Solving systems of equations (16) and (17), we obtain

$$
\begin{aligned}
& \left\{\begin{array}{l}
a_{1}(x)=\frac{(\gamma-1)\left((p(x)-1) p_{1}(x)-\gamma p_{2}(x)\right)}{p(x) p_{1}(x)+p_{2}(x)(1-\gamma)}, \\
b_{1}(x)=\frac{p(x)\left((p(x)-1) p_{1}(x)-\gamma p_{2}(x)\right)}{p(x) p_{1}(x)+p_{2}(x)(1-\gamma)}, \\
c_{1}(x)=\frac{p(x) p_{1}(x)+p_{2}(x)(1-\gamma)}{(p(x)-1) p_{1}(x)-\gamma p_{2}(x)}
\end{array}\right. \\
& \left\{\begin{array}{l}
a_{2}(x)=\frac{(\gamma-1)\left((q(x)-1) q_{1}(x)-\gamma q_{2}(x)\right)}{q(x) q_{1}(x)+q_{2}(x)(1-\gamma)}, \\
b_{2}(x)=\frac{q(x)\left((q(x)-1) q_{1}(x)-\gamma q_{2}(x)\right)}{q(x) q_{1}(x)+q_{2}(x)(1-\gamma)}, \\
c_{2}(x)=\frac{q(x) q_{1}(x)+q_{2}(x)(1-\gamma)}{(q(x)-1) q_{1}(x)-\gamma q_{2}(x)}
\end{array}\right.
\end{aligned}
$$


We substitute (18) and (19) into (14) and (15) and we arrive at the representations:

$$
\begin{aligned}
u^{\gamma}|D u|^{p(x)-1}= & u^{\frac{(\gamma-1)\left((p(x)-1) p_{1}(x)-\gamma p_{2}(x)\right)}{p(x) p_{1}(x)+p_{2}(x)(1-\gamma)}}|D u|^{\frac{p(x)\left((p(x)-1) p_{1}(x)-\gamma p_{2}(x)\right)}{p(x) p_{1}(x)+p_{2}(x)(1-\gamma)}} \varphi_{\eta}^{\frac{(p(x)-1) p_{1}(x)-\gamma p_{2}(x)}{p(x) p_{1}(x)+p_{2}(x)(1-\gamma)}} \\
& \cdot u^{\frac{p_{1}(x)(p(x)+\gamma-1)}{p(x) p_{1}(x)+p_{2}(x)(1-\gamma)}|D u|^{\frac{p_{2}(x)(p(x)+\gamma-1)}{p(x) p_{1}(x)+p_{2}(x)(1-\gamma)}} \varphi_{\eta}^{-\frac{(p(x)-1) p_{1}(x)-\gamma p_{2}(x)}{p(x) p_{1}(x)+p_{2}(x)(1-\gamma)}}} \\
v^{\gamma}|D v|^{q(x)-1}= & v^{\frac{(\gamma-1)\left((q(x)-1) q_{1}(x)-\gamma q_{2}(x)\right)}{q(x) q_{1}(x)+q_{2}(x)(1-\gamma)}}|D v|^{\frac{q(x)\left((q(x)-1) q_{1}(x)-\gamma q_{2}(x)\right)}{q(x) q_{1}(x)+q_{2}(x)(1-\gamma)}} \varphi_{\eta}^{\frac{(q(x)-1) q_{1}(x)-\gamma q_{2}(x)}{q(x) q_{1}(x)+q_{2}(x)(1-\gamma)}} \\
& \cdot v^{\frac{q_{1}(x)(q(x)+\gamma-1)}{q(x) q_{1}(x)+q_{2}(x)(1-\gamma)}}|D v|^{\frac{q_{2}(x)(q(x)+\gamma-1)}{q(x) q_{1}(x)+q_{2}(x)(1-\gamma)}} \varphi_{\eta}^{-\frac{(q(x)-1) q_{1}(x)-\gamma q_{2}(x)}{q(x) q_{1}(x)+q_{2}(x)(1-\gamma)}}
\end{aligned}
$$

We observe that as $\gamma=0$,

$$
c_{1}(x)=\frac{q(x) q_{1}(x)+q_{2}(x)}{(q(x)-1) q_{1}(x)}>\frac{(q(x)-1) q_{1}(x)+q_{2}(x)}{(q(x)-1) q_{1}(x)}=1+\frac{q_{2}(x)}{(q(x)-1) q_{1}(x)} \geqslant c_{1,0}>1
$$

and similar, $c_{2}(x) \geqslant c_{2,0}>1$. This is why the same inequalities $c_{1}(x)>1$ and $c_{2}(x)>1$ hold by the continuity for sufficiently small $|\gamma|$. Thus, applying the parametric Young inequality to the right hand sides in (12) and (13) with the exponents $c_{1}(x)$ and $c_{2}(x)$ in (18) and (19), respectively, we arrive at

$$
\begin{aligned}
\int \rho^{-\alpha}(x) v^{q_{1}(x)}|D v|^{q_{2}(x)} u^{\gamma} \varphi_{\eta} d x & +\frac{|\gamma|}{2} \int u^{\gamma-1}|D u|^{p(x)} \varphi_{\eta} d x \\
& \leqslant c_{\gamma} \int u^{\frac{p_{1}(x)(p(x)+\gamma-1)}{p_{1}(x)+p_{2}(x)}}|D u|^{\frac{p_{2}(x)(p(x)+\gamma-1)}{p_{1}(x)+p_{2}(x)}} \frac{\left|D \varphi_{\eta}\right|^{\frac{p(x) p_{1}(x)+p_{2}(x)(1-\gamma)}{p_{1}(x)+p_{2}(x)}}}{\varphi_{\eta}^{\frac{p(x) p_{1}(x)+p_{2}(x)(1-\gamma)}{p_{1}(x)+p_{2}(x)}-1}} d x \\
\int \rho^{-\beta}(x) u^{p_{1}(x)}|D u|^{p_{2}(x)} v^{\gamma} \varphi_{\eta} d x & +\frac{|\gamma|}{2} \int v^{\gamma-1}|D v|^{q(x)} \varphi_{\eta} d x \\
& \leqslant d_{\gamma} \int v^{\frac{q_{1}(x)(q+\gamma-1)}{q_{1}(x)+q_{2}(x)}}|D v|^{\frac{q_{2}(x)(q(x)+\gamma-1)}{q_{1}(x)+q_{2}(x)}} \frac{\left|D \varphi_{\eta}\right|^{\frac{q(x) q_{1}(x)+q_{2}(x)(1-\gamma)}{q_{1}(x)+q_{2}(x)}}}{\varphi_{\eta}^{\frac{q(x) q_{1}(x)+q_{2}(x)(1-\gamma)}{q_{1}(x)+q_{2}(x)}-1}} d x
\end{aligned}
$$

where the constants $c_{\gamma}$ and $d_{\gamma}$ depend only on $p(x), q(x), p_{1}(x), q_{1}(x), p_{2}(x), q_{2}(x)$ and $\gamma$. We observe that under our assumptions for $\gamma=0$ we have

$$
d_{1}(x)=\frac{p_{1}(x)+p_{2}(x)}{p(x)-1} \geqslant d_{1,0}>1, \quad d_{2}(x)=\frac{q_{1}(x)+q_{2}(x)}{q(x)-1} \geqslant d_{2,0}>1
$$

and this is why by the continuity $d_{1}(x)>1$ and $d_{2}(x)>1$ for sufficiently small $|\gamma|$. Applying the Young inequality with the exponents

$$
d_{1}(x)=\frac{p_{1}(x)+p_{2}(x)}{p(x)+\gamma-1}, \quad d_{1}^{\prime}(x)=\frac{p_{1}(x)+p_{2}(x)}{p_{1}(x)+p_{2}(x)-p(x)-\gamma+1}
$$

and

we obtain

$$
d_{2}(x)=\frac{q_{1}(x)+q_{2}(x)}{q(x)+\gamma-1}, \quad d_{2}^{\prime}(x)=\frac{q_{1}(x)+q_{2}(x)}{q_{1}(x)+q_{2}(x)-q(x)-\gamma+1},
$$

$$
\begin{aligned}
\int \rho^{-\alpha}(x) v^{q_{1}(x)}|D v|^{q_{2}(x)} u^{\gamma} \varphi_{\eta} d x+ & \frac{|\gamma|}{2} \int u^{\gamma-1}|D u|^{p(x)} \varphi_{\eta} d x \\
\leqslant & e_{\gamma} \int \rho^{-\beta}(x) u^{p_{1}(x)}|D u|^{p_{2}(x)} \varphi_{\eta} d x \\
& +f_{\gamma} \int \rho^{\frac{\beta(p(x)+\gamma-1)}{p_{1}(x)+p_{2}(x)-p(x)-\gamma+1}}(x) \frac{\left|D \varphi_{\eta}\right|^{\frac{p(x) p_{1}(x)+p_{2}(x)(1-\gamma)}{p_{1}(x)+p_{2}(x)-p(x)-\gamma+1}}}{\varphi_{\eta}^{\frac{p(x) p_{1}(x)+p_{2}(x)(1-\gamma)}{p_{1}(x)+p_{2}(x)-p(x)-\gamma+1}-1}} d x
\end{aligned}
$$




$$
\begin{aligned}
\int \rho^{-\beta}(x) u^{p_{1}(x)}|D u|^{p_{2}(x)} v^{\gamma} \varphi_{\eta} d x & +\frac{|\gamma|}{2} \int v^{\gamma-1}|D v|^{q(x)} \varphi_{\eta} d x \\
\leqslant & g_{\gamma} \int \rho^{-\alpha}(x) v^{q_{1}(x)}|D v|^{q_{2}(x)} \varphi_{\eta} d x \\
& \quad+h_{\gamma} \int \rho^{\frac{\alpha(q(x)+\gamma-1)}{q_{1}(x)+q_{2}(x)-q(x)-\gamma+1}}(x) \frac{\mid D \varphi_{\eta} \frac{q(x) q_{1}(x)+q_{2}(x)(1-\gamma)}{q_{1}(x)+q_{2}(x)-q(x)-\gamma+1}}{\varphi_{\eta}^{\frac{q(x) q_{1}(x)+q_{2}(x)(1-\gamma)}{q_{1}(x)+q_{2}(x)-q(x)-\gamma+1}-1}} d x .
\end{aligned}
$$

Employing the test functions $\psi_{1}(x)=\psi_{2}(x)=\varphi_{\eta}$ in $(10)$, we get

$$
\begin{aligned}
& \int \rho^{-\alpha}(x) v^{q_{1}(x)}|D v|^{q_{2}(x)} \varphi_{\eta} d x \leqslant \int|D u|^{p(x)-1}\left|D \varphi_{\eta}\right| d x, \\
& \int \rho^{-\beta}(x) u^{p_{1}(x)}|D u|^{p_{2}(x)} \varphi_{\eta} d x \leqslant \int|D v|^{q(x)-1}\left|D \varphi_{\eta}\right| d x .
\end{aligned}
$$

We employ the representation

$$
\begin{gathered}
|D u|^{p(x)-1}=u^{a_{3}(x)}|D u|^{b_{3}(x)} \varphi_{\eta}^{\frac{1}{c_{3}(x)}} u^{-a_{3}(x)}|D u|^{p(x)-1-b_{3}(x)}\left(\rho^{-\beta} \varphi_{\eta}\right)^{\frac{1}{d_{3}(x)}} \rho^{\frac{\beta}{d_{3}(x)}} \varphi_{\eta}^{-\frac{1}{c_{3}(x)}-\frac{1}{d_{3}(x)}}, \\
|D v|^{q(x)-1}=v^{a_{4}(x)}|D v|^{b_{4}(x)} \varphi_{\eta}^{\frac{1}{c_{4}(x)}} v^{-a_{4}(x)}|D v|^{q(x)-1-b_{4}(x)}\left(\rho^{-\alpha} \varphi_{\eta}\right)^{\frac{1}{d_{4}(x)}} \rho^{\frac{\alpha}{d_{4}(x)}} \varphi_{\eta}^{-\frac{1}{c_{4}(x)}-\frac{1}{d_{4}(x)}},
\end{gathered}
$$

in order to apply the triple Young inequality to the right hand sides in $(22)$ and $(23)$ with the exponents denoted respectively $c_{3}(x), d_{3}(x), e_{3}(x)$ and $c_{4}(x), d_{4}(x), e_{4}(x)$. Here we choose the parameters so that

$$
\begin{aligned}
& \left\{\begin{array}{l}
a_{3}(x) c_{3}(x)=\gamma-1, \\
b_{3}(x) c_{3}(x)=p(x) \\
a_{3}(x) d_{3}(x)=-p_{1}(x) \\
\left(p(x)-1-b_{3}(x)\right) d_{3}(x)=p_{2}(x), \\
\frac{1}{c_{3}(x)}+\frac{1}{d_{3}(x)}+\frac{1}{e_{3}(x)}=1
\end{array}\right. \\
& \left\{\begin{array}{l}
a_{4}(x) c_{4}(x)=\gamma-1 \\
b_{4}(x) c_{4}(x)=q(x) \\
a_{4}(x) d_{4}(x)=-q_{1}(x) \\
\left(q(x)-1-b_{4}(x)\right) d_{4}(x)=q_{2}(x) \\
\frac{1}{c_{4}(x)}+\frac{1}{d_{4}(x)}+\frac{1}{e_{4}(x)}=1
\end{array}\right.
\end{aligned}
$$

Solving systems of equations (26) and (27), we obtain

$$
\left\{\begin{aligned}
a_{3}(x) & =\frac{(\gamma-1) p_{1}(x)(p(x)-1)}{p(x) p_{1}(x)+p_{2}(x)(1-\gamma)} \\
b_{3}(x) & =\frac{p(x) p_{1}(x)(p(x)-1)}{p(x) p_{1}(x)+p_{2}(x)(1-\gamma)} \\
c_{3}(x) & =\frac{p(x) p_{1}(x)+p_{2}(x)(1-\gamma)}{p_{1}(x)(p(x)-1)} \\
d_{3}(x) & =\frac{p(x) p_{1}(x)+p_{2}(x)(1-\gamma)}{(p(x)-1)(1-\gamma)} \\
e_{3}(x) & =\frac{p(x) p_{1}(x)+p_{2}(x)(1-\gamma)}{p_{1}(x)+\left(p_{2}(x)-p+1\right)(1-\gamma)}
\end{aligned}\right.
$$




$$
\left\{\begin{array}{l}
a_{4}(x)=\frac{(\gamma-1) q_{1}(x)(q(x)-1)}{q(x) q_{1}(x)+q_{2}(x)(1-\gamma)} \\
b_{4}(x)=\frac{q(x) q_{1}(x)(q(x)-1)}{q(x) q_{1}(x)+q_{2}(x)(1-\gamma)} \\
c_{4}(x)=\frac{q(x) q_{1}(x)+q_{2}(x)(1-\gamma)}{q_{1}(x)(q(x)-1)} \\
d_{4}(x)=\frac{q(x) q_{1}(x)+q_{2}(x)(1-\gamma)}{(q(x)-1)(1-\gamma)} \\
e_{4}(x)=\frac{q(x) q_{1}(x)+q_{2}(x)(1-\gamma)}{q_{1}(x)+\left(q_{2}(x)-q+1\right)(1-\gamma)}
\end{array}\right.
$$

We observe that as $\gamma=0$

$$
\begin{aligned}
& c_{3}(x)=\frac{p(x) p_{1}(x)+p_{2}(x)}{p_{1}(x)(p(x)-1)}=1+\frac{p_{1}(x)+p_{2}(x)}{p_{1}(x)(p(x)-1)} \geqslant c_{3,0}>1, \\
& d_{3}(x)=\frac{p(x) p_{1}(x)+p_{2}(x)}{p(x)-1}=p_{1}(x)+\frac{p_{1}(x)+p_{2}(x)}{p(x)-1}>p_{1}(x) \geqslant d_{3,0}>1, \\
& e_{3}(x)=\frac{p(x) p_{1}(x)+p_{2}(x)}{p_{1}(x)+p_{2}(x)-p(x)+1}>\frac{p_{1}(x)+p_{2}(x)}{p_{1}(x)+p_{2}(x)-p(x)+1} \geqslant e_{3,0}>1
\end{aligned}
$$

and similar estimates hold for $c_{4}(x), d_{4}(x), e_{4}(x)$. Hence, the continuity implies that for sufficiently small $|\gamma|$ all these exponents exceed 1 similarly to the above arguing.

Substituting (28) and (29) into (24) and (25), we obtain the representations

$$
\begin{aligned}
& |D u|^{p(x)-1}=u^{\frac{(\gamma-1) p_{1}(x)(p(x)-1)}{p(x) p_{1}(x)+p_{2}(x)(1-\gamma)}}|D u|^{\frac{p(x) p_{1}(x)(p(x)-1)}{p(x) p_{1}(x)+p_{2}(x)(1-\gamma)}} \varphi_{\eta}^{\frac{p_{1}(x)(p(x)-1)}{p(x) p_{1}(x)+p_{2}(x)(1-\gamma)}} \\
& \cdot u^{\frac{p_{1}(x)(p(x)-1)(1-\gamma)}{p(x) p_{1}(x)+p_{2}(x)(1-\gamma)}}|D u|^{\frac{p_{2}(x)(p(x)-1)(1-\gamma)}{p(x) p_{1}(x)+p_{2}(x)(1-\gamma)}}\left(\rho^{-\beta} \varphi_{\eta}\right)^{\frac{(p(x)-1)(1-\gamma)}{p(x) p_{1}(x)+p_{2}(x)(1-\gamma)}} \\
& \cdot \rho^{\frac{\beta(p(x)-1)(1-\gamma)}{p(x) p_{1}(x)+p_{2}(x)(1-\gamma)}} \varphi_{\eta}^{\frac{\left(\gamma-p_{1}(x)-1\right)(p(x)-1)}{p(x) p_{1}(x)+p_{2}(x)(1-\gamma)}} \\
& |D v|^{q(x)-1}=v^{\frac{(\gamma-1) q_{1}(x)(q(x)-1)}{q(x) q_{1}(x)+q_{2}(x)(1-\gamma)}}|D v|^{\frac{q(x) q_{1}(x)(q(x)-1)}{q(x) q_{1}(x)+q_{2}(x)(1-\gamma)}} \varphi_{\eta}^{\frac{q_{1}(x)(x(x)-1)}{q(x) q_{1}(x)+q_{2}(x)(1-\gamma)}} \\
& \cdot v^{\frac{q_{1}(x)(q(x)-1)(1-\gamma)}{q(x) q_{1}(x)+q_{2}(x)(1-\gamma)}}|D|^{\frac{q_{2}(x)(q(x)-1)(1-\gamma)}{q(x) q_{1}(x)+q_{2}(x)(1-\gamma)}}\left(\rho^{-\alpha} \varphi_{\eta}\right)^{\frac{(q(x)-1)(1-\gamma)}{q(x) q_{1}(x)+q_{2}(x)(1-\gamma)}} \\
& \cdot \rho^{\frac{\alpha(q(x)-1)(1-\gamma)}{q(x) q_{1}(x)+q_{2}(x)(1-\gamma)}} \varphi_{\eta}^{\frac{\left(\gamma-q_{1}(x)-1\right)(q(x)-1)}{q(x) q_{1}(x)+q_{2}(x)(1-\gamma)}}
\end{aligned}
$$

and applying the triply Young inequality to the right hand sides in (22) and (23) with the exponents $c_{3}(x), d_{3}(x), e_{3}(x), c_{4}(x), d_{4}(x), e_{4}(x)$ in (28), (29) respectively, we arrive at

$$
\begin{aligned}
\int \rho^{-\alpha}(x) v^{q_{1}(x)}|D v|^{q_{2}(x)} \varphi_{\eta} d x & \\
\leqslant & C_{1} \int u^{\gamma-1}|D u|^{p(x)} \varphi_{\eta} d x+C_{2} \int \rho^{-\beta}(x) u^{p_{1}(x)}|D u|^{p_{2}(x)} \varphi_{\eta} d x \\
& +C_{3} \int \rho^{\frac{\beta(p(x)-1)(1-\gamma)}{p_{1}(x)+\left(p_{2}(x)-p+1\right)(1-\gamma)}}(x) \frac{\left|D \varphi_{\eta}\right|^{\frac{p(x) p_{1}(x)+p_{2}(x)(1-\gamma)}{p_{1}(x)+\left(p_{2}(x)-p+1\right)(1-\gamma)}}}{{\frac{p(x) p_{1}(x)+p_{2}(x)(1-\gamma)}{p_{1}(x)+\left(p_{2}(x)-p+1\right)(1-\gamma)}}^{p_{\eta}}} d x
\end{aligned}
$$




$$
\begin{aligned}
\int \rho^{-\beta}(x) u^{p_{1}(x)}|D u|^{p_{2}(x)} \varphi_{\eta} d x & \\
\leqslant & C_{4} \int v^{\gamma-1}|D v|^{q(x)} \varphi_{\eta} d x+C_{5} \int \rho^{-\alpha}(x) v^{q_{1}(x)}|D v|^{q_{2}(x)} \varphi_{\eta} d x \\
& +C_{6} \int \rho^{\frac{\alpha(q(x)-1)(1-\gamma)}{q_{1}(x)+\left(q_{2}(x)-q+1\right)(1-\gamma)}} \frac{\left|D \varphi_{\eta}\right|^{\frac{q(x) q_{1}(x)+q_{2}(x)(1-\gamma)}{q_{1}(x)+\left(q_{2}(x)-q+1\right)(1-\gamma)}}}{\varphi_{\eta}^{\frac{q(x) q_{1}(x)+q_{2}(x)(1-\gamma)}{q_{1}(x)+\left(q_{2}(x)-q+1\right)(1-\gamma)}-1}} d x .
\end{aligned}
$$

Employing (20) and (21), by the previous estimates we obtain

$$
\begin{aligned}
& \int \rho^{-\alpha}(x) v^{q_{1}(x)} \mid\left.D v\right|^{q_{2}(x)} \varphi_{\eta} d x \leqslant C_{7} \int \rho^{-\beta}(x) u^{p_{1}(x)}|D u|^{p_{2}(x)} \varphi_{\eta} d x \\
&+C_{8} \int \frac{\rho^{\frac{\beta(p(x)+\gamma-1)}{p_{1}(x)+p_{2}(x)-p(x)-\gamma+1}}(x)\left|D \varphi_{\eta}\right|^{\frac{p(x) p_{1}(x)+p_{2}(x)(1-\gamma)}{p_{1}(x)+p_{2}(x)-p(x)-\gamma+1}}}{\varphi_{\eta}^{\frac{p(x) p_{1}(x)+p_{2}(x)(1-\gamma)}{p_{1}(x)+p_{2}(x)-p(x)-\gamma+1}-1}} d x \\
&+C_{9} \int \rho^{\frac{\beta(p(x)-1)(1-\gamma)}{p_{1}(x)+\left(p_{2}(x)-p(x)+1\right)(1-\gamma)}}(x) \frac{\left|D \varphi_{\eta}\right|^{\frac{p(x) p_{1}(x)+p_{2}(x)(1-\gamma)}{p_{1}(x)+\left(p_{2}(x)-p(x)+1\right)(1-\gamma)}}}{\varphi_{\eta}^{\frac{p(x) p_{1}(x)+p_{2}(x)(1-\gamma)}{p_{1}(x)+\left(p_{2}(x)-p(x)+1\right)(1-\gamma)}}-1} \\
& \int \rho^{-\beta}(x) u^{p_{1}(x)}|D u|^{p_{2}(x)} \varphi_{\eta} d x \leqslant C_{10} \int \rho^{-\alpha}(x) v^{q_{1}(x)}|D v|^{q_{2}(x)} \varphi_{\eta} d x \\
&+C_{11} \int \frac{\rho^{\frac{\alpha(q(x)+\gamma-1)}{q_{1}(x)+q_{2}(x)-q(x)-\gamma+1}}(x)\left|D \varphi_{\eta}\right|^{\frac{q(x) q_{1}(x)+q_{2}(x)(1-\gamma)}{q_{1}(x)+q_{2}(x)-q(x)-\gamma+1}}}{\varphi_{\eta}^{\frac{q(x) q_{1}(x)+q_{2}(x)(1-\gamma)}{q_{1}(x)+q_{2}(x)-q(x)-\gamma+1}-1}} d x \\
&+C_{12} \int \rho^{\frac{\alpha(q(x)-1)(1-\gamma)}{q_{1}(x)+\left(q_{2}(x)-q(x)+1\right)(1-\gamma)}}(x) \frac{\left|D \varphi_{\eta}\right|^{\frac{q(x) q_{1}(x)+q_{2}(x)(1-\gamma)}{q_{1}(x)+\left(q_{2}(x)-q(x)+1\right)(1-\gamma)}}}{\varphi_{\eta}^{\frac{q(x) q_{1}(x)+q_{2}(x)(1-\gamma)}{q_{1}(x)+\left(q_{2}(x)-q(x)+1\right)(1-\gamma)}}-1}
\end{aligned}
$$

where the constants depend only on $p(x), q(x), p_{1}(x), q_{1}(x), p_{2}(x), q_{2}(x), \gamma$ and on the parameters in the Young inequalities.

We substitute (34) into (35) and vice versa and move all the terms of form $c \int \rho^{-\alpha}(x) v^{q_{1}(x)}|D v|^{q_{2}(x)} \varphi_{\eta} d x$ and $c \int \rho^{-\beta}(x) u^{p_{1}(x)}|D u|^{p_{2}(x)} \varphi_{\eta} d x$ into the left hand side. Then by (6) and (7), for $C_{7} C_{10}<1$ (which can be ensured by an appropriate choice of the parameters in the Young inequalities) we have

$$
\begin{aligned}
& \int \rho^{-\alpha}(x) v^{q_{1}(x)}|D v|^{q_{2}(x)} \varphi_{\eta} d x \leqslant c \sum_{j=1}^{4} D_{j, \gamma}(\eta), \\
& \int \rho^{-\beta}(x) u^{p_{1}(x)}|D u|^{p_{2}(x)} \varphi_{\eta} d x \leqslant c \sum_{j=1}^{4} D_{j, \gamma}(\eta) .
\end{aligned}
$$

Passing to the limit as $\eta \rightarrow 0_{+}$, by (11) we arrive at the contradiction. This completes the proof of the theorem.

\section{BIBLIOGRAPHY}

1. H. Brezis, X. Cabré. Some simple nonlinear PDE's without solutions // Boll. Un. Mat. Ital. B: Artic. Ric. Mat. Ser. 8. 1:2, 223-262 (1998).

2. A. Farina, J. Serrin. Entire solutions of completely coercive quasilinear elliptic equations // J. Diff. Eq. 250:12, 4367-4408 (2011).

3. A. Farina, J. Serrin Entire solutions of completely coercive quasilinear elliptic equations II // J. Diff. Eq. 250:12, 4409-4436 (2011). 
4. R. Filippucci, P. Pucci, M. Rigoli. Nonlinear weighted p-Laplacian elliptic inequalities with gradient terms // Comm. Cont. Math. 12:3, 501-535 (2010).

5. E. Galakhov, O. Salieva. On blow-up of solutions to differential inequalities with singularities on unbounded sets // J. Math. Anal. Appl. 408:1, 102-113 (2013).

6. E.I. Galakhov, O.A. Salieva. Blow-up of solutions of some nonlinear inequalities with singularities on unbounded sets // Matem. Zametki. 98:2, 187-195 (2015). [Math. Notes. 98:2, 222-229 (2015).]

7. X. Li, F. Li. Nonexistence of solutions for singular quasilinear differential inequalities with a gradient nonlinearity // Nonl. Anal. Theor. Methods. Appl. Ser. A. 75:2, 2812-2822 (2012).

8. E. Mitidieri, S.I. Pokhozhaev A priori estimates and blow-up of solutions to nonlinear partial differential equations and inequalities // Trudy Matem. Inst. Steklova. 234, 3-383 (2001). [Proc. Steklov Inst. Math. 234, 1-362 (2001).]

9. S.I. Pokhozhaev. The essentially nonlinear capacities induced by differential operators // Dokl. RAN. 357:5, 592-594 (1997). [Dokl. Math. 56:3, 924-926 (1997).]

Evgenii Igorevich Galakhov,

Peoples' Friendship University of Russia,

Miklukho-Maklaya str. 6,

117198, Moscow, Russia

E-mail: galakhov@rambler.ru

Olga Alexeevna Salieva,

Moscow State University of Technology "STANKIN",

Vadkovskii lane, 3a,

127055, Moscow, Russia

E-mail: olga.a.salieva@gmail.com 